\title{
El trabajo de soporte a los estudiantes en una escuela de bachillerato en México
}

\author{
O trabalho de apoio aos estudantes numa escola de \\ ensino médio no México
}

\section{The support work for students in a high school in Mexico}

\author{
Rita de Cássia de Souza \\ Universidade Federal de Viçosa (UFV), Viçosa/MG - Brasil
}

\section{Resumen}

Este artículo es parte de una investigación cualitativa y descriptiva hecha en una escuela de bachillerato vinculada a una universidad en México. El aporte teórico para este trabajo es la perspectiva construccionista social y se ha realizado una investigación relacional, a partir de algunos momentos de convivencia en actividades escolares, consulta a documentos y conversaciones con alumnos y profesores. El objetivo es presentar la red de apoyo material y de salud física y emocional que ayuda al estudiante a mantenerse en la escuela. Para que esto sea posible, la escuela tiene una planificación y un currículum especial, pero destaco aquí tres servicios que me parecieron fundamentales para el apoyo que la escuela ofrece a sus estudiantes y a la comunidad donde se sitúa: el CAE - Centro de Atención al Cliente Estudiante, el sector de Tutoría, Orientación y Consejo Educativo y el área de Responsabilidad Social.

Palabras clave: Bachillerato, Asistencia al educando, Acción comunitaria

\section{Resumo}

Este artigo é parte de uma pesquisa qualitativa e descritiva feita em uma escola de ensino médio vinculada a uma universidade no México. O aporte teórico para este trabalho é a perspectiva construcionista social. Foi feita uma investigação relacional, a partir de alguns momentos de convivência em atividades escolares, consulta a documentos e conversações com alunos e professores. O objetivo é apresentar a rede de apoio material e de saúde física e emocional que ajuda o estudante a se manter na escola e concluir seus estudos. Para que isso seja possível, a escola tem um planejamento e um currículo especial, mas destaco aqui três serviços que me pareceram fundamentais para o apoio que a escola oferece aos seus estudantes e à comunidade onde se situa: o CAE - Centro de Atenção ao Estudante, o setor de Tutoria, Orientação e Conselho Educativo e a área de Responsabilidade Social.

Palavras-chave: Ensino médio, Assistência ao educando, Ação comunitária

\section{Abstract}

This article is part of a qualitative and descriptive research carried out at a high school linked to a university in Mexico. The theoretical input to this work is the Social Constructionist perspective. We carried out a relational investigation, from some moments of interaction in school activities, consultation of documents and 
conversations with students and teachers. The objective is to present a physical and emotional health and physical support network that helps students to stay in school and complete their studies. To make this possible, the school has a planning and a special curriculum, and here are three fundamental services for the support the school offers to its students and to the community where it is located: CAE - Center of Attention to the Student, the Tutoring, Orientation and Educational Council sector, and the Social Responsibility area.

Keywords: High school, Assistance to the student, Community action

\section{Introducción}

Este artículo es parte de una investigación ${ }^{1}$ que hice en la UABIC, Unidad Académica Bachillerato con Interacción Comunitaria, escuela de bachillerato en la ciudad de Mérida, México, vinculada a la UADY² - Universidad Autónoma de Yucatán, una universidad pública ${ }^{3}$ de la provincia de Yucatán. Mi intención es presentar un trabajo innovador hecho por esta escuela en relación a su dedicación a los estudiantes, a la formación que imparte, a las aportaciones que pretende llevar a cabo en la sociedad, cambiando la historia de vida de un grupo de jóvenes y adolescentes de una comunidad de bajos recursos de México.

Esta investigación es cualitativa y descriptiva hecha a partir de un período de convivencia en la escuela, donde estuve algunos días durante los meses de septiembre y octubre de 2018, asistiendo a clases y otras actividades como la Jornada de Ciencia, Cultura y Tecnología y conversando con profesores y estudiantes.

\footnotetext{
${ }^{1}$ Este artículo es parte de una investigación de posdoctorado hecha en el Instituto Kanankil en la ciudad de Mérida, México. Agradezco las aportaciones de María del Rocío Chaveste Gutierrez y Papusa - María Luisa Molina López por las conversaciones tan agradables y de mucho aprendizaje. Agradezco a Laura Cámara Bauserman por su lectura y corrección del español. Dedico este artículo a la Uabic y agradezco al coordinador administrativo Guillermo Contreras Gil, por la confianza en permitir la investigación y la publicación del artículo con el nombre de la institución, a todos los profesores que cedieron su tiempo e informaciones de su trabajo y a los estudiantes que me recibieron en sus clases y me ayudaron a comprender mejor cómo funciona la escuela.

2 Para más informaciones: http://www.uady.mx/. En México, las universidades son responsabilidad de los Estados y el Gobierno Federal tiene solamente una universidad que se Ilama Unam Universidad Nacional Autónoma de México.

${ }^{3}$ En México, las instituciones públicas no son gratuitas, pero los estudiantes pagan menos que en las instituciones privadas de enseñanza. Según Mitchel, los estudiantes pagan cerca de 250 pesos para inscribirse en el examen de Ceneval, pero, una vez aprobados, no pagan más para estudiar. Nos informó que, hasta el año pasado, los estudiantes recibieron una camisa de uniforme y podrían comprar otra, si lo quisieran. También pueden usar camisa blanca o azul para ir a la escuela. Patricia me dijo que los maestros no usan libros didácticos y preparan sus materiales con copias que les salen más baratas a los estudiantes.
} 
El aporte teórico para este trabajo es la perspectiva Construccionista Social o "perspectivas construccionistas", dado su carácter heterogéneo (SPINK; MEDRADO; MÉLLO, 2014). Para Vivien Burr (2015), la publicación del artículo "Psicología Social como historia", en 1973, marca la emergencia del Construccionismo Social cuando el autor, Kenneth Gergen defiende que la psicología social tiene características radicalmente diferentes de las ciencias naturales, por ejemplo: los resultados obtenidos en las investigaciones de la psicología social pueden ser comunicados a las personas y éstas, a su vez, pueden cambiar su manera de actuar.

A la luz de los argumentos presentes, el continuo intento de construir leyes generales de comportamiento social parece estar mal encaminado, y la creencia asociada de que el conocimiento de la interacción social pueda acumularse de una manera similar al de las ciencias naturales parece injustificada. En esencia, el estudio de la psicología social es principalmente una tarea histórica. Nos encontramos esencialmente involucrados en un recuento sistemático de los asuntos contemporáneos. Usamos la metodología científica, pero los resultados nos son principios científicos en el sentido tradicional. (GERGEN, 1988, p. 43)

Para el autor, "Los fenómenos sociales pueden cambiar considerablemente en la medida en que se encuentran sujetos al cambio histórico." (GERGEN, 1988, p. 44). Sin embargo, para Kenneth y Mary Gergen, "La construcción social no es atribuible a un único individuo ni a un grupo, y tampoco es singular ni unificada, sino que responde a una creación compartida socialmente." (GERGEN; GERGEN; 2011, p.9).

El construccionismo social se opone a la psicología tradicional que comprende al ser humano como individualizado y con una esencia interior (BURR, 2015). Para el construccionismo, no hay un yo interior, los seres humanos son intrínsecamente relacionales, dependientes de los otros y del medio. No es una perspectiva determinista, el ser relacional interacción es dinámico y en constante construcción que involucra al individuo, a sus pares y al ambiente donde vive. La identidad de cada uno es siempre relacional, o sea, negociada y dependiente de los demás. Como también, la realidad en la que vivimos es fruto de una constante negociación de sentidos en los que el lenguaje tiene un carácter, no de representar el mundo sino de crearlo. (GERGEN, 2016). Esa concepción de ser relacional tiene muchas implicaciones para la comprensión del mundo y, por supuesto, para el trabajo de investigación. 
Las investigaciones construccionistas sociales se oponen a las empiristas por: creer que no hay métodos mejores para llegar a la verdad, porque no hay una verdad a ser descubierta y considerar que todo es un producto de una construcción social, incluso los métodos científicos. Por eso, la elección del método tiene que ver con el contenido que se desea generar en la investigación. Como no es posible una investigación neutral y sin ningún tipo de valoración, el investigador debe tener conciencia de los valores que sustenta su trabajo y que la investigación puede generar. Las investigaciones de base construccionista se centran en lo que las personas hacen juntas y, por esto, hay un esfuerzo por involucrar a los participantes, que denomino y considero colaboradores, en todas las partes del trabajo, haciendo la investigación con ellos y no sobre ellos. En la siguiente tabla aparece un resumen de las diferencias entre los dos tipos de investigación:

Cuadro 1: Diferencias entre las investigaciones empíricas y construccionistas sociales

\begin{tabular}{cc}
\hline Empirismo & Social Construcción \\
\hline Observación & Interpretación/ Construcción \\
\hline Valor neutral & Valor creado \\
\hline Métodos objetivos & Prácticas de apoyo \\
\hline Validez & Consenso \\
\hline Verdad & Utilidad social \\
\hline Estadísticas & Descripción rica
\end{tabular}

Fuente: GERGEN, 2015, p. 66 (traducción nuestra)

Partiendo de esos principios, hice una investigación relacional y orientada al proceso (MCNAMEE, 2015):

Al considerar la investigación como un proceso relacional de creación de significado y comprensión de manera colaborativa, nos damos cuenta de que todos los relatos son descripciones simultáneas de eventos y parte del evento en sí, debido a la naturaleza co-constructiva de la interacción. [...] Ninguna investigación puede o puede proporcionar un resultado definitivo. Todo el conocimiento es provisional y cuestionable (de alguna otra comunidad lingüística). Todos los relatos son local, histórico y culturalmente específicos ${ }^{4}$. (MCNAMEE, 2014, p. 93)

Harlene Anderson (2012) propone algunos principios básicos para crear un contexto de colaboración y conversación dialógica propuestos por (2012). Aunque la autora estuviera hablando del trabajo de un terapeuta, yo los utilicé por considerar

\footnotetext{
4 "In viewing research as a relational process of crafting meaning and understanding collaboratively, we become more mindful that all accounts are simultaneously descriptions of events and part of the event itself, due to the co-constructive nature or talk, of interaction. No research will or can provide the definitive result. All knowledge is provisional and contestable (from some other language community). All accounts are locally, historically and culturally specific." (MCNAMEE, 2014, p. 93)
} 
que son muy útiles al oficio del investigador que quiere hacer una investigación relacional, colaborativa y dialógica.

La postura filosófica tiene siete rasgos definidos e interrelacionados que sirven como sensibilidades que orientan la acción y la forma de ser del terapeuta: indagación mutua, expertise relacional, no-saber, ser abierto, vivir con incertidumbre, transformación mutua y orientación hasta la vida cotidiana común. (ANDERSON, 2012, p. 9)

Después de obtener el permiso para hacer la investigación en la escuela, empecé por hacer un seguimiento de algunas clases de estudiantes del $3^{\text {er }}$ año de bachillerato y, los maestros o el director me indicaban a alguien que podría contestarme algunas preguntas sobre el trabajo de la escuela. Fue así que llegué a los siguientes colaboradores que aceptaron, a su vez, participar en la investigación ${ }^{5}:$ 1) Jesús Bibian Puc Tun, psicólogo y responsable del CAE - Centro de Atención al Estudiante; 2) Mitchel Antonio Guerrero González, licenciado en Educación, maestro 6 fundador de la Uabic, fue, de 2009 a 2015 coordinador del área de comunicación y, desde 2015, es el gestor académico 7 ; 3) Diana Marisol Rodríguez Bastos, psicóloga y responsable por el área de Tutoría, Orientación y Consejo Educativo y también fundadora de la escuela; 4) Patricia del Rosario Baas Rejón, licenciada en Ciencias de la Familia, con una especialidad en Docencia, cursaba, en 2018, una Maestría en Psicología Educativa. Es la coordinadora del área de Responsabilidad Social de la Uabic.

Siguiendo los principios presentados por Anderson (2012), invité a los colaboradores para una conversación individual sobre su trabajo en la escuela. Todos los diálogos fueron en la escuela en el horario de disponibilidad de cada uno. A pesar de la existencia de una grabadora y de la intencionalidad de la investigación, intenté promover una interacción lo más próxima posible de una conversación cotidiana, con apertura para que indagasen sobre sus dudas y

\footnotetext{
${ }^{5}$ Todos los colaboradores fueron invitados a participar y aceptaron grabar sus relatos. Las fotos también fueron hechas con el consentimiento de la escuela. El artículo fue enviado a todos los que participaron en la investigación y al director de la escuela para su aprobación y su publicación tenía que ser previamente aceptada por ellos.

${ }^{6}$ Mitchel imparte clases de Redacción de textos y Organización de la Información y siempre tiene un grupo de clase: "para que pueda vivir en la realidad y verlo directamente lo que me comentan los alumnos y los maestros." (Mitchel, en conversación, 2018)

7 El gestor académico coordina a los coordinadores de las áreas de Matemáticas, de Ciencias Naturales, de Ciencias Sociales, Deportes y Tecnologías.
} 
curiosidades, pero, obviamente, yo tenía mucho más qué preguntar pues quería conocer las actividades de la escuela.

En general, cuando tenía la aprobación para conectar la grabadora ${ }^{8}$, empezaba las conversaciones solicitando a los colaboradores que dijeran su nombre, cómo empezaron a trabajar en la escuela, cuál era su función y las características de su trabajo y, así, seguía el flujo evitando interrumpir o dirigir el diálogo. Como era la primera vez que hacía diálogos con fines de investigación sin usar un guion, tenía miedo de no obtener nada significativo o no saber qué preguntar. Pero, como había convivido en las clases y actividades, leído algunos documentos, tenía muchas dudas y curiosidad acerca de varios aspectos de la escuela. Fue importante también la postura de no-saber, pues consideraba todo el tiempo que no debía presuponer o interpretar nada y siempre preguntar, buscando saber el punto de vista de los colaboradores. La expertise relacional me ayudó a percibir que mi tarea era conducir la investigación promoviendo las conversaciones, transcribiendo, produciendo informaciones sobre la escuela junto con los colaboradores, que son los expertos en el tema, los que conocen a fondo el cotidiano escolar y la colaboración de todos fue esencial para la producción del trabajo.

Todas las conversaciones fueron tranquilas y, cada una, con más o menos una hora de duración. Los colaboradores pudieron reflexionar sobre su trabajo, sobre todo lo que habían hecho hasta ahora en la escuela, los logros y los retos que tienen. En cuanto a mí, además del crecimiento personal por conocer un poco de la historia de cada uno, las conversaciones me hicieron creer aún más en el poder de transformación de la escuela para la vida de las personas, de una comunidad y de toda una sociedad.

\section{La escuela y los servicios prestados a los estudiantes y la comunidad}

Antes de la construcción de la Uabic, la Uady, además de la oferta de educación superior, tenía 2 escuelas de bachillerato ${ }^{9}$, conocidas como prepas

\footnotetext{
${ }^{8}$ Usé una aplicación de smartphone para hacer las grabaciones. Las transcripciones son muy fieles al original, pero algunas repeticiones y vicios de lenguaje fueron retirados.

${ }^{9}$ La Escuela Preparatoria Un (conocida como Prepa 1) fue fundada junto a la propia Uady en 1922, la Escuela Preparatoria Dos (Prepa 2) fue oficialmente creada en octubre de 1977. Por eso, llaman la Uabic de Prepa 3. Según Mitchel, otras escuelas pueden vincularse a la Uady. Para eso, pagan por los derechos, implementan el Plan de Estudios de la Uady y se les llama Prepas incorporadas
} 
(abreviatura de preparatoria), que son muy bien calificadas y preparan a sus estudiantes para una formación universitaria, en general, en la propia Uady, considerada la mejor universidad del Estado. Para estudiar en una de estas escuelas, es necesario aprobar un examen de selección bastante riguroso. La Uabic, también conocida como prepa 3 , es más reciente y fue creada con características diferentes de las dos otras prepas de la Universidad. La región donde está ubicada la escuela fue especialmente elegida justamente por las dificultades económicas y sociales de su población, como nos cuenta la historia de la escuela:

La Universidad Autónoma de Yucatán (Uady) en su carácter de institución pública y siguiendo el modelo humanista que la caracteriza, se propone desarrollar socialmente a las personas que viven en condiciones de marginación social y económica, aportar las condiciones académicas para disminuir el rezago social, educativo y económico, además de ampliar su cobertura educativa en el Nivel Medio Superior a través de la creación de un Programa Educativo (PE) de Bachillerato, dirigido a las personas de comunidades de Yucatán cuyas condiciones socioeconómicas de vulnerabilidad, les dificultan continuar con su preparación académica en este nivel.

Las necesidades sociales de la región, el crecimiento demográfico y la falta de espacios físicos para el número de solicitantes que, año con año demandan ingreso al bachillerato de nuestra Universidad, son algunas de las razones que llevaron a forjar y mantener el compromiso de la Uady para diseñar e implementar un programa educativo que promueva y desarrolle competencias en los alumnos con el reflejo de la calidad que la caracteriza y le permita promover la mejora en el nivel social de la población y su calidad de vida. (HISTORIA UABIC)

Hasta 2018, en la zona donde está ubicada, la Uabic es la única preparatoria que prepara a los estudiantes para la educación superior, pues las demás son técnicas. El hecho de ser Unidad Académica significa que, en el futuro, esta misma institución puede albergar programas de capacitación o de formación para la vida, o incluso formación superior, con Maestría y Doctorado. En el momento, la escuela tiene el bachillerato y, los sábados, algunos profesores de la Uady imparten la asignatura El Profesional y la Comunidad para estudiantes de licenciaturas como Psicología, Matemáticas y Diseño del hábitat y hacen actividades prácticas: a veces los estudiantes salen para atender a la comunidad y a veces la gente vienen a la escuela como, por ejemplo, para atención psicológica.

a la Uady. Hay 51 escuelas preparatorias particulares incorporadas a la Uady en la ciudad de Mérida y en Yucatán. 
En la Uabic, hay una estructura que involucra a los estudiantes en actividades socio-comunitarias ${ }^{10}$ del primer al último año de bachillerato. Es un modelo único, creado por la Uady y que las otras dos prepas no manejan. Esto porque, según Patricia, fue un modelo diseñado como una respuesta a un diagnóstico hecho por la universidad, antes de construir la escuela, acerca de las problemáticas y características de la comunidad. Los resultados indicaron: problemas delictivos, desintegración familiar, asentamientos irregulares, alcoholismo y pobreza. Con estos dados, la Universidad planeó un programa de bachillerato en que los alumnos son agentes de cambio para modificar su entorno, haciendo acciones muy concretas en la comunidad.

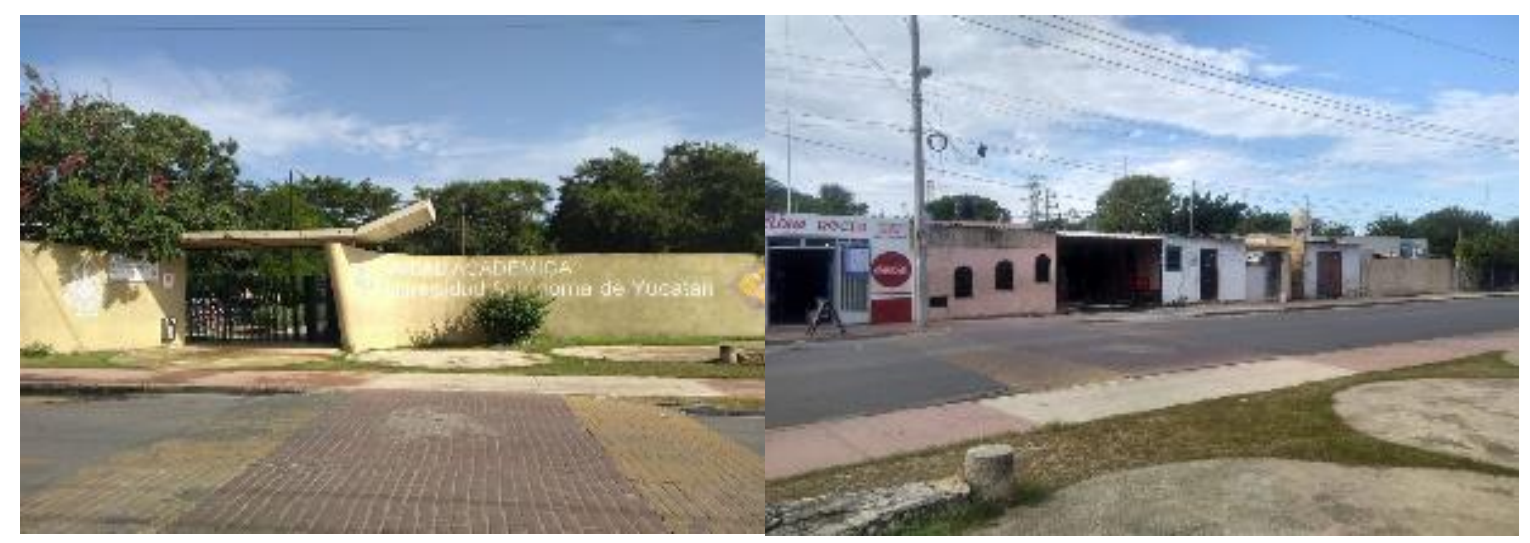

Figura 1. Foto de la entrada principal de la escuela Figura 2. La vista de la entrada principal del barrio $^{11}$

La escuela empezó sus actividades en septiembre de 2009 con 206 alumnos divididos en 8 grupos y, en 2018, tenía 877 estudiantes distribuidos en 25 grupos (9 de primero, 8 de segundo, 8 de tercero) y 58 profesores (6 de Ciencias Naturales, 4 de Informática, 7 de Comunicación, 12 de Desarrollo Físico, Artístico y Cultural, 7 de Responsabilidad Social, 6 de Matemáticas, 11 de Ciencias Sociales y Humanidades y 4 de Desarrollo Humano Integral) ${ }^{12}$. El gestor académico nos dijo

\footnotetext{
${ }^{10}$ Para eso, la Uady creó el Modelo Institucional de Responsabilidad Social Universitaria (Mirsu) con el objetivo de: "articular de manera estratégica las acciones de Responsabilidad Social que permitan la traducción de los deberes éticos en términos de gestión institucional, con la finalidad de impactar de manera positiva en el contexto social, económico y ambiental de la Universidad, de la región y del país". Recuperado de: http://www.dgplanei.uady.mx/rsu.php. el 30 de octubre de 2018.

${ }^{11}$ Todas las fotos las hizo la investigadora en 2018 y su publicación fue autorizada por la escuela.

12 Algunas personas son contratadas para hacer algunos trabajos, pero no son maestros de la escuela. Estuve, por ejemplo, con dos personas que hacían un seguimiento de los trabajos socio comunitarios de los estudiantes y se les contrataba solamente para esta actividad.
} 
que, en 2018, 500 jóvenes se presentaron al examen y que entran alrededor de 300 estudiantes al año.

Según Diana y Mitchel, cuando empezó la unidad, para la primera generación, se revisaron bases de datos de la Secretaría de Educación Pública acerca de egresados de secundaria y personas de la Uady fueron a la zona a invitar a los estudiantes a estudiar, pero llegaron algunos que no querían o no tenían comprometimiento con los estudios. "A veces teníamos aquí chicos que tenían dinero y tenían mucha facilidad económica y se destacaban de los demás de alguna manera. Era muy evidente." (Mitchel, en conversación, 2018).

Al año siguiente, 2010, se establecieron dos criterios de selección que se verificaban de forma paralela: el examen Exani $I^{13}(49 \%)$ y un estudio socioeconómico (51\%) que debía comprobar que el aspirante era de bajos recursos ${ }^{14}$. El resultado era que el nivel socioeconómico se imponía a los resultados del examen e ingresaban estudiantes de muy bajos recursos, pero que no necesariamente tenían buenos resultados del Exani I $^{15}$. Por eso, en 2018, hubo un cambio en el momento en el que se verifican los dos criterios: el estudio socioeconómico se realiza previo al Exani I. Solamente los aspirantes que reflejan el nivel de recursos bajos siguen en el proceso de selección y presentan el Exani I e ingresan los alumnos con mejores resultados en el examen. "Entonces ahora

\footnotetext{
${ }^{13}$ El Exani-I lo organiza el Ceneval - Centro Nacional de Evaluación para la Educación Superior, asociación civil cuya actividad principal es el diseño y la aplicación de instrumentos de evaluación de conocimientos, habilidades y competencias. "El Exani-I es un examen que ofrece información para identificar a los aspirantes con mayor aptitud para lograr un buen desempeño en el primer ciclo escolar de la educación media superior. Se integra al aspirante con dos exámenes: Exani-I Admisión, que es un instrumento estandarizado de aptitud académica que evalúa conocimientos y habilidades numéricas, verbales y no verbales en áreas de la comunicación y pensamiento matemático que son predictivas del desempeño académico en el primer ciclo escolar. Exani-I Diagnóstico, que explora conocimientos y habilidades en áreas disciplinares de la educación básica para identificar el logro académico. Es un examen de diagnóstico y la institución tiene la opción de incluirlo o no en su proceso de admisión. Verifica en la convocatoria o en servicios escolares de la institución que elegiste si lo debes presentar." Recuperado de: http://www.ceneval.edu.mx/exani-i. el 28 de noviembre de 2018. Para hacer el examen, los estudiantes pagan por la inscripción más 0 menos 250 pesos mexicanos.

${ }^{14}$ Conversando con los estudiantes de $3^{\text {er }}$ grado, me dijeron que, al registrarse para el examen de selección, rellenaron un cuestionario sobre su situación económica y, que, una vez aprobados en la selección, todos ellos recibieron una visita de personas de la Uady en sus casas buscando comprobar si situación económica se correspondía con lo registrado. Según Michel, son personas del Departamento de Educación Media Superior de la Uady que realizan las visitas a las casas para averiguar las condiciones socioeconómicas de los estudiantes.

${ }^{15}$ Diana nos contó un caso específico en el que un estudiante llegó con un diagnóstico de déficit cognitivo y no lograba buenas calificaciones y, después de muchas juntas con la familia, la decisión conjunta fue transferirlo a una escuela técnica de la región.
} 
solamente ingresan alumnos que lo necesitan económicamente, pero con buenos resultados" (Mitchel, en conversación, 2018).

Una vez en la escuela, los estudiantes tienen acceso, además de las actividades escolares, a una serie de apoyos del punto de vista de salud física y mental, económico y de informaciones que les ayuda a mantenerse estudiando y concluir el bachillerato.

La escuela tiene una consulta de Odontología y una de Nutrición ${ }^{16}$ que recibe estudiantes que están haciendo prácticas en la Uady o servicios sociales ${ }^{17}$. El protocolo de la atención implica conocer la historia clínica, hacer un diagnóstico, proponer un plan de tratamiento y ejecutarlo hasta que el consultante termine de alta. El odontólogo y el nutriólogo tienen su propia agenda y van poniendo a sus pacientes. La indicación es que reciban a los estudiantes cuando ellos no tengan clase, pero cuando no es posible, hay un documento para que el estudiante pueda salir de clase para ir a la consulta, siempre que no sean clases de matemáticas ni de inglés. Hay también un servicio de enfermería disponible durante todos los días lectivos del año:

Es una enfermera que está aquí de base, o sea, forma parte de la unidad y el objetivo que tiene el consultorio de enfermería es atender los síntomas de los estudiantes. Es decir, para evitar que el estudiante se vaya a casa y pierda clases, primero pasa por la enfermería para ver si realmente necesita ir a casa o necesita ir a consultar. Cuando sienten un dolor de cabeza, o es algo de la presión, los estudiantes pasan por la enfermería y también hay ciertos estudiantes que tienen alguna condición médica que constantemente tienen que estar monitoreando signos vitales. (Bibian, en conversación, 2018)

${ }^{16}$ Conversé con dos estudiantes de Nutrición de la Uady que estaban haciendo prácticas en la escuela y me dijeron que se hizo un diagnóstico general con los estudiantes de la escuela para detectar problemas y, a partir de ahí, crearon un Proyecto de Intervención entre los estudiantes. Además, también les atienden en consultas personales. Con excepción para las emergencias, los estudiantes menores de edad necesitan el consentimiento firmado por un responsable para una atención en las dos consultas.

17 En México, todo el estudiante de nivel superior, especialmente si estudió en una universidad pública, tiene que hacer servicio social, o sea, cuando termina su formación hace un semestre de trabajo gratuito (con supervisión de profesores en la universidad) en comunidades de bajas condiciones económicas y, sólo después de cumplir este trabajo, puede solicitar su diploma. 


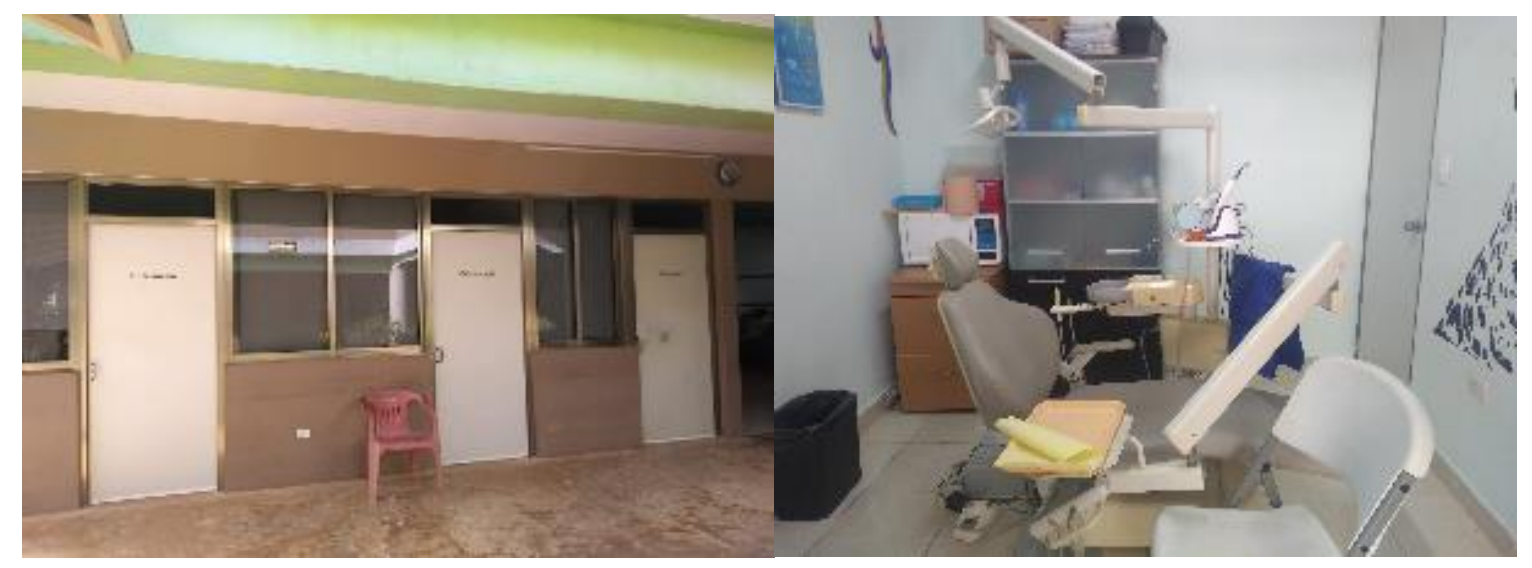

Figura 3. Consultorios de enfermería, nutrición y odontología Figura 4. Consultorios de odontología

Cuando se matriculan en la Uabic, los estudiantes pasan a tener acceso al seguro social del IMSS - Instituto Mexicano del Seguro Social. Según Bibian, hace poco, la Presidencia de la República de México dictaminó que todas las universidades tenían que asegurar que sus estudiantes estuvieran afiliados ${ }^{18}$ :

Entonces ya nos obligaron como tal a ver que todos hagan el trámite. Es muy sencillo, esa reforma trajo consigo acercar y digitalizar los procedimientos. Los estudiantes entran en una liga de Internet, generan su número de seguro social y nos traen a nosotros como CAE y nosotros solicitamos esta alta que tarda entre 2 a 3 días y los estudiantes ingresan en otro link para escoger su clínica, su unidad médica familiar cercana y ya nosotros les pedimos su cartilla y con eso pueden consultar. El seguro de estudiante incluye operaciones, maternidad, pues es el más amplio que existe en el sistema mexicano. Es un derecho, es gratuito y no hay ningún costo de por medio. (Bibian, en conversación, 2018)

La Uabic tiene un Centro de Atención al Estudiante (CAE) que presta varios servicios a los estudiantes. Incluso desde antes de su ingreso en la escuela, el CAE da informaciones sobre las inscripciones realizadas en internet en una fecha concreta.

Ellos [los adolescentes que quieren participar de la selección] vienen con nosotros, nos preguntan sobre el proceso de selección, les explicamos del principio al fin el del proceso. Hay quien no tiene acceso a Internet, a una computadora. Vienen con nosotros y aquí hacen el registro, les tomamos su fotografía para que puedan subir al sistema y solucionamos dudas en forma general. (Bibian, en conversación, 2018)

A los estudiantes que ya están en la escuela, el CAE les presta artículos que olvidaron en casa o no pudieron comprar, como calculadoras científicas,

\footnotetext{
${ }^{18}$ En México, el estudiante puede tener el IMSS hasta los 25 años y después, tiene que acceder a otro modelo de seguro social donde hay que una aportación económica para acceder a los servicios.
} 
diccionarios, batas de laboratorio, hilos y agujas para coser su ropa, playeras, libretas y juegos para distraerse en las horas de recreo. Los alumnos también pueden usar las computadoras del espacio y de la biblioteca para hacer tareas escolares. Según Bibian, los estudiantes dejan su tarjeta de identificación para coger los materiales y, cuando los devuelven, recuperan su carné.

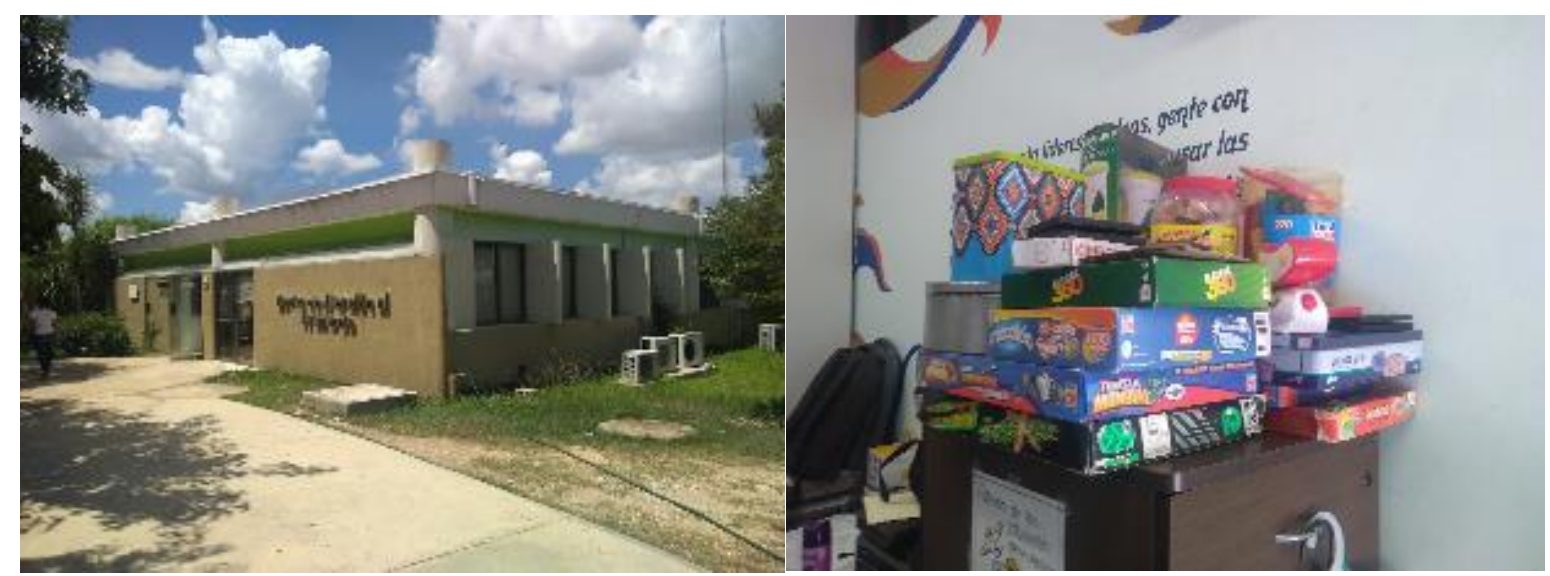

Figura 5. CAE Centro de Atención al Estudiante

Figura 6. Juegos que los estudiantes utilizan en su tiempo libre en la escuela.

De todas las actividades del CAE, tal vez la más importante para los estudiantes sea la ayuda para obtener becas ${ }^{19}$ desde las de transporte ${ }^{20}$ y apoyo económico, hasta las de excelencia, artísticas y deportivas ${ }^{21}$.

Aquí en la Uabic, las que se solicitan más son por cuestiones económicas. La mayoría de los estudiantes de acá tienen beca que ya traen desde siempre, que se llama Prospera ${ }^{22}$. Entonces de una u otra forma, el estudiante cuenta con algún tipo

${ }^{19}$ El Plan de Gobierno del nuevo presidente de México, Andrés Manuel López Obrador (AMLO), prevé la concesión de una beca de 800 pesos mensuales a todos los estudiantes de preparatorias públicas en el país. Recuperado de: https://lopezobrador.org.mx/?s=becas. el 28 de noviembre de 2018.

${ }^{20}$ La beca de transporte consiste en un apoyo monetario de $\$ 800.00$ pesos bimestrales, con una duración de hasta 4 meses.

${ }^{21}$ Las becas pueden ser: 1) Cbsems (Coordinación de Becas de la Subsecretaría de Educación Media Superior) de la Subsecretaría de Educación Media Superior (SEMS); 2) Abogado Francisco Repetto Milán que es una beca propia de la Uady que es la que se maneja más en la Uabic, pero hay también de 3) Ibecey, del Instituto de Becas y Crédito Educativo del Estado de Yucatán y del 4) Ayuntamiento de Mérida. "En el caso del lbecey que es del Estatal y del ayuntamiento que es municipal ellos becan a la familia. Es decir, una vez que algún miembro de la familia tenga algún recurso, ya nadie más de la familia lo puede tener. Entonces de ahí se ve que, si hay algún estudiante que tiene hermanitos en la primaria, secundaria o en otras prepas 0 en licenciatura prefiere no escoger esa beca, porque si alguien escoge esta beca, ya nadie más de la casa puede tenerla. Los estudiantes prefieren buscar las becas que becan a estudiantes y no a la familia Además de que el lbecey y el Ayuntamiento, en comparación con los pagos que hacen las otras becas, es muy poco lo que pagan" (Bibian, en conversación, 2018).

${ }^{22}$ Prospera es una beca del Gobierno de México que ayuda económicamente a familias de bajos recursos financieros. No son para estudiantes sino para familias. 
de apoyo o recurso. Es un poco difícil, por lo menos acá, que alguien solicite excelencia. Ha habido estudiantes que tienen 100 de promedio, pero, como las reglas te piden mantener el 100 al término de semestre, entonces eso desanima un poco a los estudiantes porque no saben si al final lo pueden mantener. Hay una beca de lbecey ${ }^{23}$ que puede ser de excelencia académica, artística o deportiva. El estudiante que tenga 100 de la modalidad académica o que haya recibido algún premio artístico, deportivo, o algún reconocimiento a nivel nacional, o a nivel estatal puede postularse para tener una beca de excelencia académica, deportiva 0 artística. Pero, acá no hemos tenido ningún postulante en ese sentido. (Bibian, en conversación, 2018).

Cada beca tiene sus reglas de operación, sus propios criterios de selección de los candidatos ${ }^{24}$, pero:

Para todas las becas te piden ser regular, o sea, no deber materias. Si debes materia, no eres candidato a obtener una beca. [...] Las becas son para cada ciclo escolar, no se renuevan automáticamente. El estudiante tiene que hacer el proceso de registro de solicitud cada año si así lo desea. (Bibian, en conversación, 2018)

De acuerdo con Bibian, entre el 50 y el $60 \%$ de los estudiantes de la escuela tienen algún tipo de beca. Además de las becas, los estudiantes de la Uabic participan en un Programa de bienestar digital del Gobierno Estatal dirigido a la población vulnerable y reciben, el primer año, una laptop en comodato. Cuando terminan el bachillerato, pueden quedarse con el aparato, pero los que dejan la escuela antes, por abandono o suspenso, tienen que devolver la computadora ${ }^{25}$.

El acceso a una computadora y a Internet es muy importante porque todas las asignaturas de la escuela tienen actividades en línea. La escuela tiene conexión a Internet para los estudiantes, pero, ésta no llega a las aulas. Las redes sociales son el principal canal de comunicación del CAE con la familia y la comunidad, a través de las que obtienen muchas informaciones sin necesidad de ir a la escuela o hacer llamadas telefónicas.

23 Ibecey - Instituto de Becas Crédito Educativo del Estado de Yucatán. Recuperado de: http://www.ibecey.yucatan.gob.mx/. 28 de noviembre de 2018

${ }^{24}$ Me llamó la atención que, para la beca de Cbsems (Coordinación de Becas de la Subsecretaría de Educación Media Superior) de la SEP (Secretaría de Educación Pública) Federal, tienen un tabulador y pagan al estudiante en función del grado escolar y del sexo. "Por ejemplo, de tercer año, a las mujeres, se les pagan 800 pesos, ya a los hombres de tercero les pagan $\$ 725$, o sea $\$ 75$ pesos más se les paga a las mujeres. Esto es porque, en las reglas de operación del programa, se dice que las mujeres, en general, como estandarización, en toda la República porque es federal, tienden a tener más gastos. Esos $\$ 75$ de diferencia hace que estén en equidad de circunstancias. $Y$ es diferente también para el primer año, el segundo... Por ejemplo, las mujeres de segundo año cobran $\$ 725$ y los hombres, $\$ 75$ menos, lo que son $\$ 650$ pesos." (Bibian, en conversación, 2018)

${ }^{25}$ Hubo un problema y no todos los estudiantes de la Uabic recibieron la laptop y siguen esperando el envío del Gobierno. 
En general creo que es una percepción de todos que el Facebook del CAE es efectivo, llega a mucha gente y así los maestros nos piden cosas para que publiquemos: por ejemplo, que van a hacer corte de cabellos, que tal día hay junta de padres. Es el principal medio de comunicación y el más efectivo en ese sentido y es lo que tiene más seguidores. [...] Entonces es otra de las características del CAE que utiliza esos lenguajes, utiliza estos medios, que ahorita los estudiantes y los papás están usando, para estar al día en las formas en cómo se comunican. (Bibian, en conversación, 2018)

De hecho, el día 30 de noviembre de 2018, la página de Facebook del CAE ${ }^{26}$ tenía 6.397 seguidores y, algunos días antes, encontré las siguientes publicaciones:
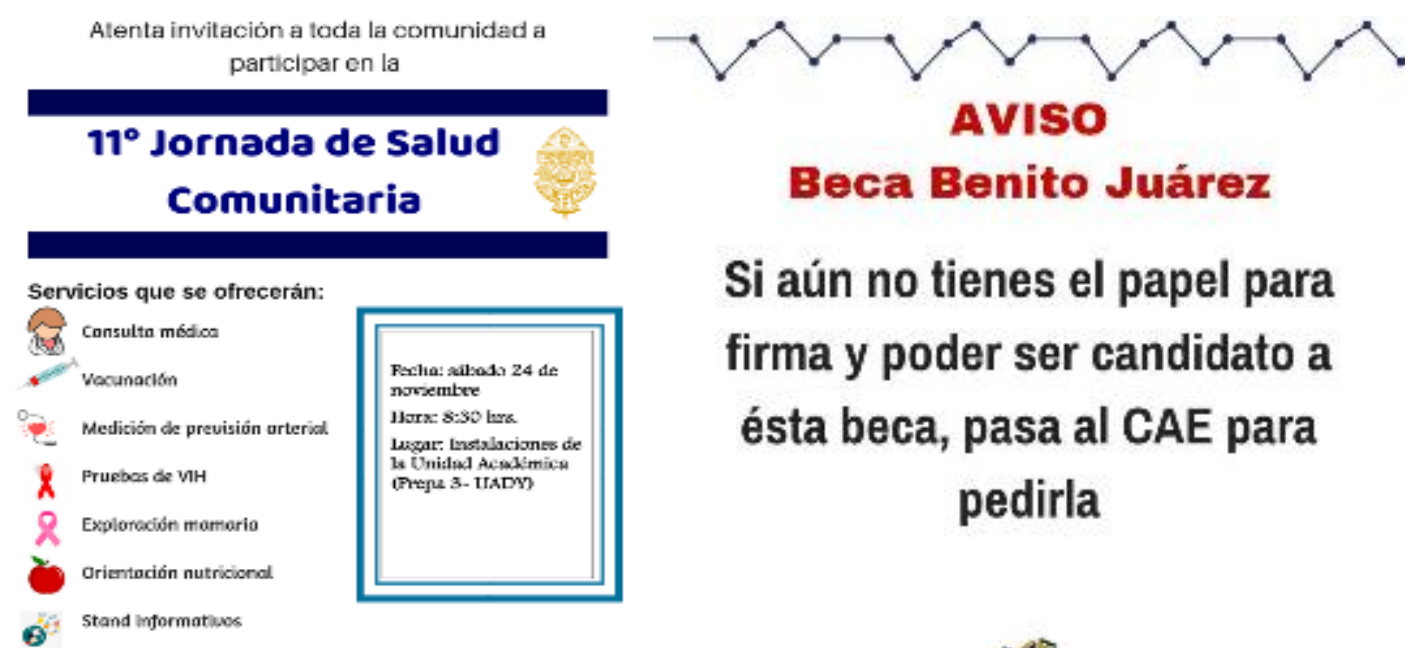
firma y poder ser candidato a ésta beca, pasa al CAE para pedirla

Asiste con to familia, evento gratuito

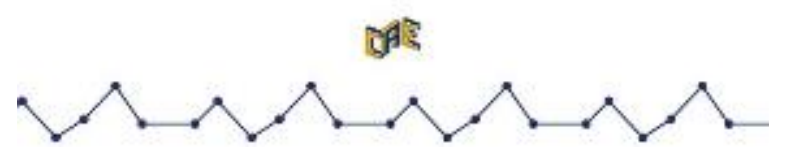

Figuras 7 y 8: Imágenes recuperadas de Facebook del CAE - UABIC el 22 de noviembre de 2018

EI CAE funciona en un espacio pequeño y, siempre que pasaba por allá, en intervalos de clase, estaba lleno de estudiantes. Además de Bibian, hay otra persona que trabaja en el CAE, y como en otros departamentos de la Uabic, tienen preferencia de trabajo las personas que se formaron en la prepa o en la Uady.

Creemos que es una oportunidad laboral de trabajo. Estamos procurando darles espacio a los que ya formamos, ya conocen la filosofía de la Uady, conocen a los estudiantes porque fueron estudiantes. Estas cuestiones hacen que el CAE sea efectivo, esta sensibilidad que tenemos, que tienen todos los que han estado, de saber que hemos estado en ese lugar. Entonces procuramos dar un servicio lo más cálido, amable y tratando de ayudar a los estudiantes que a veces vienen

26 CAE - Facebook. Recuperado de: https://www.facebook.com/pg/caeuabicuady/community/?ref=page internal. 30 de noviembre de 2018. Facebook es una página de Internet que funciona como una red para conectar virtualmente personas, grupos y asociaciones en diversas partes del mundo. 
desesperados y aquí les resolvemos en la medida que podamos. Las personas que han estado en el CAE han sido estudiantes egresados de acá. Estamos dando oportunidad laboral a los que hemos formado por 3 años. Hay una convocatoria, hay un proceso de selección y se selecciona a los que mejor vayan con los criterios que pedimos. (Bibian, en conversación, 2018)

Lo que dice Bibian del trabajo del CAE nos permite ver la importancia del servicio que prestan a los estudiantes:

Somos un punto de referencia. El estudiante tiene la confianza de venir a preguntar cosas que ni siquiera son parte de la escuela. Una vez han preguntado: ¿cómo saco un pasaporte?, ¿dónde puedo ir a buscar un trabajo? Esto es producto de la confianza que hemos generado. En lo general, los estudiantes pueden venir a preguntar cosas de lo que sea, de los extraordinarios, juntas, dónde está tal maestro, o tal área. [...] En el CAE creemos que el estudiante no sólo es estudiante, sino es integral, es persona, es hijo, es alguien. No basta nada más con saciar las cuestiones académicas, sino también las cuestiones de salud, para eso están los consultorios y las cosas personales. Con el hecho que tengan un espacio para venir y convivir personal también estamos ayudando a la formación integral del estudiante. (Bibian, en conversación, 2018)

Bibian nos informa que, cada fin de semestre, los estudiantes tienen que evaluar a los maestros y demás departamentos de la escuela a través de un instrumento donde dicen cómo son atendidos, si están resolviendo sus necesidades, si son rápidos, si tienen alguna sugerencia o felicitación y, acerca del trabajo del CAE: "En la mayoría de las veces, hemos salido bien calificados" (Bibian, en conversación, 2018).

Además del CAE, la escuela tiene un servicio de Tutoría, Orientación y Consejo con 6 tutores, siendo 5 psicólogos (uno con Maestría en Psicología Criminológica y dos con Maestría en Psicoterapia) y una persona licenciada en educación con una Maestría en Consejo Educativo. Ellos ayudan a los estudiantes en sus dificultades escolares y personales, evitando la reprobación y el abandono escolar. Por ejemplo, cuando el estudiante no es aprobado en una asignatura y tiene que pasar por un recursamiento, es obligatorio que sea acompañado por un tutor que lo ayuda a organizar sus horarios, planear horas de estudio y encontrar mejores técnicas de aprendizaje y maneras de sanar sus dudas en relación al contenido.

Los tutores también supervisan semanalmente la asistencia a clase y siguen el Programa "Yo no Abandono", creado por la SEP - Secretaría de Educación Pública de México: 
El Movimiento contra el Abandono Escolar es una estrategia integral de carácter nacional que involucra a la participación conjunta y coordinada de autoridades educativas, federales y estatales, directivos de planteles, docentes, padres de familia, estudiantes y sociedad en general, para lograr mayores índices de acceso, permanencia y conclusión exitosa de los estudios de nivel medio superior. (YO no abandono)

A partir del Programa, hay un seguimiento individual y personal de los estudiantes que están faltando a clase para saber lo que pasa, informar y pedir apoyo a la familia lo más pronto posible, evitando que la situación se agrave y el estudiante deje de estudiar.

\begin{abstract}
Una de las cosas que hacemos como parte de "Yo no abandono" es que desde hace ya casi tres años, se coloca, en salones, unas listas de inasistencia. Siempre les pedimos a los profesores para registrar cuando el estudiante no llega. Entonces cada semana nosotros lo verificamos. Eso nos ha permitido acercarnos mucho a los estudiantes porque ellos ya saben: "me van a llamar si yo no vine". Generalmente si el alumno dejó de venir lo llamamos y si no llegamos a vernos, llamamos al papá. Entonces es un mecanismo muy bueno para la parte de prevención y también para involucrar a los papás, para que lo sepan, porque a veces, según los papás, dejan a su hijo y no lo saben. Esta actividad ha sido muy enriquecedora para nosotros. (Diana, en conversación, 2018)
\end{abstract}

Además de este trabajo, el equipo de psicología realiza asistencia médica para ayudar a los estudiantes en casos de dificultades escolares o personales. Cuando hay necesidad de atención médica a los menores de 18 años, la familia es informada y tiene que firmar un documento de consentimiento. Se permiten hasta 12 sesiones y, en el caso de que el estudiante necesite más, se le dirige a otras instancias que lo hacen gratuitamente.

Los tutores también imparten asignaturas semanales de Formación Humana todos los semestres del bachillerato. Cada una tiene 32 horas semestrales, siendo 16 presenciales y 16 virtuales. Los semestres impares son más para autoconocimiento y los semestres pares manejan más la parte vocacional. Son las siguientes asignaturas: 1) Adaptación en contextos; 2) Construyendo mi futuro; 3) Relaciones Humanas Efectivas; 4) Proyecto de Carrera; 5) Relaciones Humanas Afectivas y 6) Proyecto de Vida ${ }^{27}$.

A partir de la lectura de los documentos y conversaciones, me resultó evidente que existe, desde la elaboración del Plan de Trabajo una preocupación

\footnotetext{
${ }^{27}$ Las asignaturas están disponibles em: PLAN de Estudios de Bachillerato General Universitario de la UADY. Julio, 2015. Recuperado de:

http://www.unidadacademica.uady.mx/userfiles/calendario/VEPLANBGU.pdf. en 28 nov. 2018
} 
con una formación integral de los estudiantes. La escuela ofrece una formación de gran calidad, exige mucha dedicación de tiempo y estudios y las exigencias para aprobar son altas, del $70 \%$ en cada asignatura. Por otro lado, hay toda una infraestructura espacial y profesional de apoyo a los estudiantes en sus necesidades. La escuela hace un trabajo colectivo y colaborativo en el sentido de ayudar a los estudiantes en todo lo posible y hay un compromiso del grupo en ofrecer todas las posibilidades para que los estudiantes terminen el bachillerato de la mejor manera. Cada estudiante importa.

Otra característica muy innovadora de la UABIC es el trabajo de responsabilidad social en el que se involucra a los estudiantes en actividades socio comunitarias promoviendo una mejoría en su entorno social. En todos los semestres, los estudiantes hacen, obligatoriamente, asignaturas de 64 horas cada (parte presencial y parte no) y, con excepción para el primer y último semestre, están implicados en proyectos sociales cerca de 1 hora y media semanalmente. En el primero, no hacen directamente las actividades socio comunitarias porque están preparándose para el trabajo, conociendo sus implicaciones y maneras de actuar. En el último, están más dedicados al término del curso y a la preparación para los exámenes para la universidad y hacen una evaluación del trabajo que realizaron. Las asignaturas que implican el trabajo social son: 1) Responsabilidad Social Universitaria; 2) Estrategias para el diálogo comunitario; 3) Diagnóstico comunitario participativo 4) Diseño de proyectos comunitarios; 5) Implementación de proyectos comunitarios y 6) Evaluación de proyectos comunitarios.

Las actividades socio comunitarias se organizan en tres áreas: 1. Cuidado del medio ambiente; 2. Educación y 3. Promoción de la salud. Los estudiantes eligen en qué área quieren trabajar y, cuando es preciso hacer una división equitativa entre los grupos, los maestros consideran los intereses de los alumnos, sus características personales y, especialmente, lo que quieren estudiar en la licenciatura o trabajar en el futuro. Además de un trabajo social, es un proceso formativo para los estudiantes pues todos ellos se integran con estudiantes de otros grupos de clase, tienen que organizar un proyecto, divulgar, hacer prácticas e interactuar con personas de fuera de la escuela, lo que les ayuda a desarrollar habilidades profesionales, sociales, de colaboración, organización y presentación. Todos los trabajos son supervisados directamente por los maestros y están siempre 
siendo evaluados por los estudiantes, por la comunidad y por los profesores, no sólo con el objetivo de puntuarlos sino, principalmente, para mejorarlos.

\section{Consideraciones finales}

En la investigación relacional, tan o más importante que los resultados, es el proceso. Nada sería posible sin la disponibilidad y dedicación de todos los colaboradores, que me permitieron hacer todo tipo de preguntas, convivir en varias actividades escolares y tener la libertad de escribir sobre la escuela presentando su nombre, fotos y la identificación de cada uno. Pienso que eso es fruto de una relación de confianza que se creó durante nuestra convivencia y revela la seguridad que tienen en su trabajo y el orgullo que sienten de exponerlo, incluso reconociendo que están en proceso de construcción de un modelo y que, a veces, valoran si es éste el camino que se debe seguir o si hay que hacer alteraciones. Al intentar responder a demandas sociales que son dinámicas, los colaboradores nos decían que estaban siempre atentos para revisar sus planes y ajustar las actividades, cuando les parecía más adecuado.

No se trata de concluir si la escuela es buena o mala, si es más adecuada o con mejor formación para los estudiantes. Cuestiones estas, incluso, bastante difíciles de medir. Si es fácil saber cuántos de los alumnos llegan a la universidad, no sería lo mismo saber cuánto les ayuda la escuela a comprometerse con los estudios, a planear lo que quieren para el futuro, a trabajar colaborativamente, a respetar a los demás, a ser más ecológicos y responsables consigo mismos y con su entorno. También no se trata de buscar los fallos de la escuela, lo que no hacen bien o de considerar que todo es perfecto y no puede haber críticas.

El objetivo de este artículo fue presentar, a partir de una mirada personal, construida con apoyo de varios colaboradores, lo que me pareció ser un trabajo muy innovador de una escuela de bachillerato que presta importantes servicios a sus estudiantes, la comunidad donde se ubica y, por consecuencia, a la sociedad en general. La Uady, además de crear la preparatoria ofreciendo una educación de calidad a los jóvenes de una zona marginada de la ciudad, desarrolló una estructura curricular y de personal que ayuda los estudiantes a concluir sus estudios, a pesar de sus dificultades económicas y de las exigencias de dedicación y compromiso de la escuela. 
Ciertamente, hay más cuestiones que deben ser abordadas en la escuela, pero elegí hablar del CAE, del trabajo del equipo de psicología y de la responsabilidad social como sectores de apoyo fundamentales, tanto para los estudiantes, como para la comunidad. El CAE, que existe en la Universidad y también en las otras prepas de la Uady, es todavía más importante para los estudiantes de la Uabic muchos de los cuales podrían no haber estudiado si no tuvieran el apoyo de becas, materiales y también informaciones fundamentales para su formación escolar, personal y profesional. El servicio que el equipo de psicología permite que, con tantos estudiantes, la escuela consiga hacer un seguimiento muy preciso de cada uno de los alumnos, o, por lo menos, de los que más necesitan apoyo escolar o personal. El trabajo de responsabilidad social, además de contribuir a la formación de los estudiantes crea una relación muy directa con la comunidad, un compromiso con la transformación social, la generación de una responsabilidad colectiva por el bien común. Creo que esto es muy importante, no porque la escuela esté en una zona de bajos recursos, sino porque hay mucho que hacer en el mundo. ¿Qué pasaría si más escuelas se involucrasen con su entorno en un trabajo colaborativo de transformación social?

Para muchos de los estudiantes, la Uabic es una oportunidad para sus vidas y sus familias, no solamente por la formación escolar, sino también por los cuidados que reciben de salud, bienestar, formación personal y profesional. Lamentablemente, no todos los estudiantes que quieren llegan a la escuela. La selección implica que la escuela tenga un grupo de estudiantes con mayor rendimiento y más condiciones de llegar a la universidad. Por otro lado, la escuela no se encierra en sus muros. El trabajo de responsabilidad social es una manera importante de llevar un poco de su formación a la comunidad y de preparar a los estudiantes comprometidos con el medio ambiente, los demás y los más vulnerables. Es una escuela que se dedica a mejorar, en todos los niveles posibles, la calidad de vida de sus estudiantes y de la población donde se ubica.

El Construccionismo Social, base teórica de esta investigación, defiende que la educación, como todas las cosas, son construcciones sociales. O sea, la escuela que tenemos es fruto de decisiones colectivas. Tendemos a naturalizar el proceso escolar como si fuera estable y poco pasible de modificaciones, pero lo que se hace hoy en la escuela no era así en el pasado y puede no ser así en el futuro. Llegando 
a sus diez años de historia, la Uabic refuerza la idea de que es posible construir nuevas maneras de hacer educación escolar, incluso en un contexto de vulnerabilidad económica, incluso manteniendo una meta de preparar estudiantes para la universidad y con exigencias de alto rendimiento por parte de los alumnos.

Promover educación escolar de calidad a una población excluida de derechos y que, muchas veces, no tiene muy claro lo que la educación escolar puede traer de beneficios para sus jóvenes es un reto. Para mí, lo que me pareció más especial en la escuela, fue, ante todo, el compromiso de las personas con los estudiantes y la comunidad. Un compromiso personal y colectivo. Un trabajo de colaboración que lograba que todos estuviesen interconectados. Todos me comentaron de reuniones donde hacen planes, conocen las dificultades de cada uno y establecen actividades de apoyo entre ellos. Es un acuerdo colectivo, renovado cotidianamente, de ayudar a cada uno de los estudiantes a llegar a su mejor versión, de construir, junto a la comunidad, una versión mejorada de sí misma: más ecológica, saludable y unida. La Uabic nos enseña su camino, pero cada escuela debe crear el suyo.

\section{Referencias}

ANDERSON, H. Collaborative Relationships and Dialogic Conversations: Ideas for a Relationally Responsive Practice. Family Process, v.51, n.1, 1-20, 2012.

BECAS para estudiantes de preparatoria en México en el Plan de Gobierno de AMLO. Recuperado de: https://lopezobrador.org.mx/?s=becas el 28 noviembre de 2018

BURR, V. Social Constructionism. 3 ed. New York: Routledge, 2015.

CAE - Facebook. Recuperado de:

https://www.facebook.com/caeuabicuady/?ref=br_rs el 30 noviembre de 2018.

CENEVAL. Centro Nacional de Evaluación para la Educación Superior. 2018.

Recuperado de: http://www.ceneval.edu.mx/ el 16 de noviembre de 2018.

EXANI-I. Recuperado de: http://www.ceneval.edu.mx/exani-i el 28 de noviembre de 2018.

GERGEN, K. J. An invitation to Social Construction. 3 ed. California: Sage Publications, 2015.

El ser relacional: más allá del yo y de la comunidad. Bilbao, España:

Desclée De Brouwer, 2016. 
GERGEN, K. J. La Psicología Social Como Historia. Revista Anthropos: Huellas del conocimiento, 177, 39-49, 1998.

; GERGEN, M. Reflexiones sobre la construcción social. Barcelona, Buenos Aires, México: Paidós, 2011.

HISTORIA UABIC. (s.f.) Recuperado de:

http://www.unidadacademica.uady.mx/historia.php

el 21 de septiembre de 2018.

IBECEY. Instituto de Becas Crédito Educativo del Estado de Yucatán.

Recuperado de: http://www.ibecey.yucatan.gob.mx/ el 28 noviembre de 2018

MCNAMEE, S. Radical presence: Alternatives to the therapeutic state. European Journal of Psychotherapy and Counseling, 17:4, 373-383, 2015.

MCNAMEE, S. Research as relational practice: explore modes of inquiry. In: Simon, G.; Chard, A. (Eds) Systemic inquiry: innovations in reflexive practice research. London: Everything is Connected Press, 2014.

MIRSU - Modelo Institucional de Responsabilidad Social Universitaria en la UADY (s.f). Recuperado de:

http://www.dgplanei.uady.mx/data/rsu/PRESENTACl\%C3\%93N\%20MODELO.PD Fel 16 noviembre 2018

PLAN DE ESTUDIOS del Bachillerato General Universitario. (2015) Recuperado de: http://www.unidadacademica.uady.mx/userfiles/calendario/VEPLANBGU.pdf

el 28 noviembre de 2018

RSU. Responsabilidad Social Universitaria (s.f.) Recuperado de:

http://www.dgplanei.uady.mx/rsu.php el 16 noviembre de 2018

SPINK, M.J.; MEDRADO, B. MÉLLO, R.P. Vinte e cinco anos nos rastros, trilhas e riscos de produções acadêmicas situadas. In: SPINK, M. J. P.; BRIGAGÃO, J. I.M.; NASCIMENTO, V. L. V. ; CORDEIRO, M. P. (ORGS). A produção de informação na pesquisa social: compartilhando ferramentas. Rio de Janeiro: Centro Edelstein de Pesquisas sociais, 2014.

UADY. Universidad Autónoma de Yucatán. (s.f) Recuperado de:

http://www.uady.mx/ el 22 noviembre de 2018.

YO no abandono. (s.f.) Recuperado de:

http://www.sems.gob.mx/es/sems/yo no abandono

el 22 noviembre de 2018. 